\title{
A drifting GPS buoy for retrieving effective riverbed bathymetry.
}

\author{
R. Hostache, ${ }^{\mathrm{a}, *}$, P. Matgen ${ }^{\mathrm{a}}$, L. Giustarini ${ }^{\mathrm{a}}$, F.N. Teferle ${ }^{\mathrm{b}}$, C. Tailliez ${ }^{\mathrm{a}}$, J.-F. Iffly ${ }^{\mathrm{a}}$, G. Corato ${ }^{\mathrm{a}}$ \\ ${ }^{a}$ CRP-Gabriel Lippmann, 41 rue du Brill, L-4422 Belvaux, Luxembourg. \\ ${ }^{b}$ University of Luxembourg, Faculté des Sciences, de la Technologie et de la Communication, 6 rue Richard Coudenhove-Kalergi, L-1359 \\ Luxembourg, Luxembourg.
}

\begin{abstract}
Spatially distributed riverbed bathymetry information are rarely available but mandatory for accurate hydrodynamic modeling. This study aims at evaluating the potential of the Global Navigation Satellite System (GNSS), like for instance Global Positioning System (GPS), for retrieving such data. Drifting buoys equipped with navigation systems such as GPS enable the quasi-continuous measurement of water surface elevation, from virtually any point in the world. The present study investigates the potential of assimilating GNSS-derived water surface elevation measurements into hydraulic models in order to retrieve effective riverbed bathymetry. First tests with a GPS dualfrequency receiver show that the root mean squared error (RMSE) on the elevation measurement equals $30 \mathrm{~cm}$ provided that a differential post processing is performed. Next, synthetic observations of a drifting buoy were generated assuming a $30 \mathrm{~cm}$ average error of water surface elevation (WSE) measurements. By assimilating the synthetic observation into a 1D-Hydrodynamic model, we show that the riverbed bathymetry can be retrieved with an accuracy of $36 \mathrm{~cm}$. Moreover, the WSEs simulated by the hydrodynamic model using the retrieved bathymetry are in good agreement with the synthetic "truth", exhibiting an RMSE of $27 \mathrm{~cm}$.
\end{abstract}

Keywords: Global Navigation Satellite System (GNSS) - Global Positioning System (GPS), Water Surface Elevation (WSE), effective bathymetry, hydrodynamic modeling, data assimilation

\section{Introduction}

The cost of damage caused by flooding is highly dependent on the warning time given before an event, making the issuing of timely flood alerts critical for minimizing the cost of flood damage. Predicting floods therefore remains a key concern of our society. Flood inundation models play a central role in real-time flood forecasting. In advanced hydro-meteorological forecasting systems, they provide information about expected flood hazard and damages. The models are used to accurately predict the timing and magnitude of a flood. The utility of any given model is, however, dependent on the availability of the necessary input data. Uncertainties in flood inundation modeling tend to be very high (Pappenberger et al., 2007), despite the physical laws that hydrodynamic models are generally based upon. This is partly a result of numerical approximations within hydrodynamic models, but it mainly derives

${ }^{*}$ Corresponding author, Email: hostache@lippmann.lu, Tel: +352 470261-417, Fax: +352 470264

1

2

Preprint submitted to J. Hydrol. from inadequate or lacking data on the geometry of the channel and the floodplain, the difficulty in estimating roughness coefficients and the uncertainty in initial and boundary conditions (Smith et al., 2009).

Channel and floodplain topography are required for setting up a hydrodynamic model. While the floodplain geometry can be extracted from freely available topography databases, it is important to mention that there is no database for the world's river bathymetries. The SRTM mission digital elevation model (DEM) for instance covers the Earth surface with a spatial resolution of $90 \mathrm{~m}$. In addition, the Tandem-X mission DEM is expected to provide, from 2014 on, a global surface coverage with a spatial resolution of $12 \mathrm{~m}$. However these data sources are known to have their inherent limitations, especially in narrow valleys and densely populated areas. More accurate elevation data sources like DEMs derived from airborne Lidar techniques can be an alternative for providing floodplain topography, but they come at a cost.

As a global database of river bathymetries does not exist, and because of the necessity to penetrate water 
for a direct measurement of bathymetry, time and cost 93 intensive field campaigns are generally required.

In this context of lacking riverbed bathymetry data, Durand et al. (2008) and Yoon et al. (2012) showed that river Water Surface Elevation (WSE) measurements from the proposed Surface Water Ocean Topography (SWOT) satellite mission should be helpful for estimating bathymetries using assimilation techniques in hy- 100 drodynamic models. Based on a Ka-band SAR interferometer, SWOT will provide gridded WSE information for inland lakes and rivers wider than $50 \mathrm{~m}$. The images provided by SWOT will have a $50 \mathrm{~m}$ spatial resol- ${ }_{104}$ ution on $120 \mathrm{~km}$ wide swath and the WSE is expected to 105 be measured with a centimeter vertical accuracy over a 106 $1 \mathrm{~km}^{2}$ area (Alsdorf et al., 2007). In the previously men- ${ }_{107}$ tioned proof-of-concept studies, synthetically generated 108 SWOT observations of WSE were assimilated into the 109 LISFLOOD-FP hydrodynamic model. Durand et al. 110 (2008) were able to estimate bathymetry in five loc- 111 ations along the Amazon river with an accuracy of 112 $56 \mathrm{~cm}$ using the ensemble Kalman filter. Yoon et al. 113 (2012) made use of the local ensemble batch smoother 114 (LEnBS) assimilation scheme and were able to estim- 115 ate the bathymetry with a $52 \mathrm{~cm}$ reach average accuracy 116 for the Ohio river after assimilating 8 virtual SWOT re- 117 visit cycles. SWOT is foreseen to be launched in 2020. 118 This paper introduces an alternative technique, based on 119 GNSS.

At present, only a limited number of research stu- 121 dies have investigated the potential of GNSS like 122 GPS for WSE measurements. GNSSs are currently 123 mostly used for monitoring sea level (Hong et al., 2008; 124 Watson et al., 2008; Bisnath et al., 2003). Bisnath et al. 125 (2003) found that real time kinematic (RTK) car- 126 rier phase is able to provide tide level estimates ${ }_{127}$ with a vertical accuracy of $10 \mathrm{~cm}$. Moreover, 128 Holtschlag and Aichele (2001) deployed drifting buoys 129 equipped with GPS in order to investigate flow patterns 130 and describe turbulent dispersion characteristics within 131 river reaches. More recently in a case study on the ${ }_{132}$ river Mekong, Apel et al. (2012) showed that moored ${ }_{133}$ GNSS equipped buoys were able to provide WSE mea- ${ }_{134}$ surements with an accuracy of $2 \mathrm{~cm}$.

In the light of these encouraging results and with the ${ }_{136}$ advent of advanced GNSS, such as Galileo, and with ${ }_{137}$ correction information (Differential GNSS) from net- 138 works of fixed stations becoming more readily available $\quad 139$ in near-real time, GNSS-supported measuring devices 140 can be considered a promising alternative for obtaining 141 WSE and flow velocities at a large number of locations. 142

Furthermore, over the last years, there has been 143 a significant progress with respect to the integration 144 of distributed hydrometric data with hydrodynamic models (e.g. Neal et al., 2007; Andreadis et al., 2007; Matgen et al., 2010; Hostache et al., 2010; Giustarini et al., 2011; Biancamaria et al., 2011).

In such data assimilation studies, modeled state variables or model parameters are sequentially verified and updated via measurements. The idea behind this is to merge the high temporal and spatial resolution of generally rather poor model predictions with more accurate but intermittent remote sensing observations to yield the best possible model simulations. Furthermore, if integrated with parameter estimation techniques, there is the potential to estimate uncertain model parameters, which may be used to increase the accuracy of the model (Montanari et al., 2009). Data assimilation techniques based on different versions of the Kalman filter have been used to assimilate ground gauge-based river level data at points along river reaches (Madsen and Skotner, 2005; Neal et al., 2007) from which discharge can be estimated through state augmentation. Despite this potential, applications of assimilation techniques with distributed stage data continue to be rare. In one of the few studies of this type, Andreadis et al. (2007) successfully used a square-root ensemble Kalman filter to assimilate synthetic WSE measurements from the proposed SWOT satellite mission with simulations from a hydrodynamic model for estimating river discharge. This study showed that the assimilation of 8 successive SWOT overpasses allowed a reduction of the relative error of discharge estimations from $23.2 \%$ to $10 \%$. Lai and Monnier (2009) and Hostache et al. (2010) applied a variational data assimilation method using distributed WSE in order to combine in an optimal way measurement data and a 2D shallow water model. This assimilation process allowed (1) the identification of optimal Manning friction coefficients and (2) the identification of areas in the floodplain and the channel where frictions are homogeneous. Smith et al. (2009) assimilated distributed data from wireless sensor networks in a parsimonious time series model to produce forecasts with reduced uncertainty. Matgen et al. (2010), and later on Giustarini et al. (2011), demonstrated the usefulness of assimilating via a particle filter WSE derived from satellite SAR images to improve flood forecasts.

In this general framework, the study aims at proposing a synthetic experiment to evaluate the benefit of assimilating GNSS-derived WSE measurements into a hydrodynamic $1 \mathrm{D}$ model for effective bathymetry retrievals. It has to be noted here that we defined effective bathymetry in relation with a hydrodynamic model. Indeed we defined in this study the effective bathymetry as the river channel geometry allowing for correctly pre- 
dicting flood propagation using a given model. As a 193 matter of fact, an effective bathymetry is defined in re- 194 lation to a given hydrodynamic model with given para- 195 meters and simplifications, but the methodology presen- 196 ted is this paper remains generic and can be applied us- 197 ing other hydrodynamic models. Moreover, during this 198 study we made use only of GPS, but the same method 199 can be applied to any other kind of GNSS.

\section{Material and methods}

This section introduces the design of a GPS buoy and proposes an assimilation technique for effective bathymetry retrievals. Moreover, it describes a synthetic experiment allowing to assess the usefulness of assimilating WSE provided by the buoy into a hydraulic model. In the context of a proof-of-concept study, the main advantage of using synthetically generated data rather than actual measurements is to allow for controlling the errors and their structure. It is important and necessary to demonstrate the efficiency of an assimilation scheme in a controlled environment before it can be applied to actual measurements. Moreover, a synthetic experiment, with known errors, facilitates the 215 identification of advantages and drawbacks of the procedure itself.

\subsection{Designing the GPS Buoy}

The aim of the GPS-equipped buoy is to provide 220 WSE measurements with sufficient accuracy, in or- 221 der to enable the retrieval of riverbed bathymetry 222 through data assimilation. Based on the evaluations of ${ }_{223}$ Hostache et al. (2009) and more recently Matgen et al. 224 (2010), we define an elevation measurement accuracy 225 of $30 \mathrm{~cm}$ as a target value for GPS-derived WSE mea- 226 surements. The system is composed of a water-proof ${ }_{227}$ canoe-box with a transparent hemispheric lid, filled 228 with an integrated dual-frequency GPS, namely the 229 Hemisphere A22 ${ }^{T M}$ Smart Antenna. The hemispheric ${ }_{230}$ lid is used to limit potential GPS signal perturbations. 231 To protect it from strong shocks during deployment and 232 to ensure its buoyancy and stability, the integrated sys- 233 tem is surrounded by a tire (Figure 1). The two fre- 234 quencies, L1/L2, of the GPS receiver allow correcting 235 the major part of the positioning errors due to the iono- 236 sphere (Kim and Tinin, 2009). In addition, a Post Pro- 237 cessing Kinematic treatment is applied to the data in or- 238 der to reduce bias and noise. For this post treatment 239 we take advantage of the Luxembourg network of per- 240 manent GNSS stations (SPSLux). These reference sta- 241 tions, with accurately known coordinates and altitudes, 242 enable the estimation of the correction parameters. The latter can be used to correct the error associated with a rover GPS receiver in differential mode, provided that the rover is not too distant from the reference station (Apel et al., 2012). In case a reference GPS station would not be available, an alternative would be to make use of OMNISTAR (Martinez et al., 2000), that offers a worldwide differential GPS service, based on reference stations, high power satellites and global network control centers (www . omnistar. com).

It is worth mentioning that a hydro-acoustic sensor, such as a sonar or Acoustic Doppler Current Profiler (ADCP) mounted on a buoy, can be considered as an alternative for obtaining riverbed bathymetry. These systems provide a means for directly measuring water depth. However, in this study we adopted an indirect retrieval technique based on GPS data that is less sensitive to the stability of the buoy and that is not impacted by the sediment concentration in the water (due to bedload in particular). These two aspects are known to significantly increase the measurement errors associated with hydro-acoustic sensors.

\subsection{Assimilating GPS derived water surface elevation}

This section presents the general framework of the assimilation scheme. More specific details related to the hydrodynamic model and the synthetic experiment are presented in section 2.3.

The aim of the assimilation technique is to exploit WSE recorded by a GPS in order to retrieve unknown bathymetry.

The data assimilation scheme applied in this study is a modified version of the Particle Filter (PF). The particle filter is an ensemble-based assimilation technique. This means that the prior probability of a state variable is estimated using a sample of model simulations (Smith et al., 2008). The PF computes posterior probabilities of state variables using a weighting procedure. In the PF, there is no need to formulate restrictive hypotheses on the model and observation density functions (Moradkhani, 2007). This is a key advantage of the PF with respect to the more widely used Ensemble Kalman Filter EnKF (e.g. Burgers et al., 1998; Evensen, 1994).

In this study, we use a variant of a PF that we propose to call particle smoother (PS) hereafter. This variant of the PF is comparable to the one proposed by Dunne and Entekhabi (2005) for the ensemble Kalman filter. This means that all observations distributed over time are assimilated at once to update the model state variables at any time step. According to Plaza et al. 


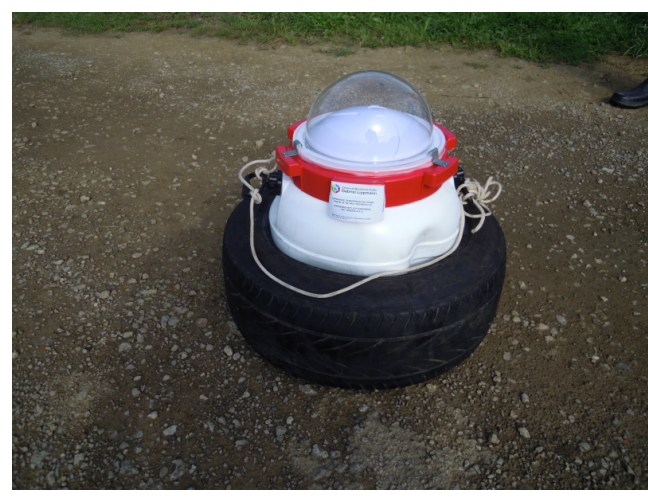

(a)

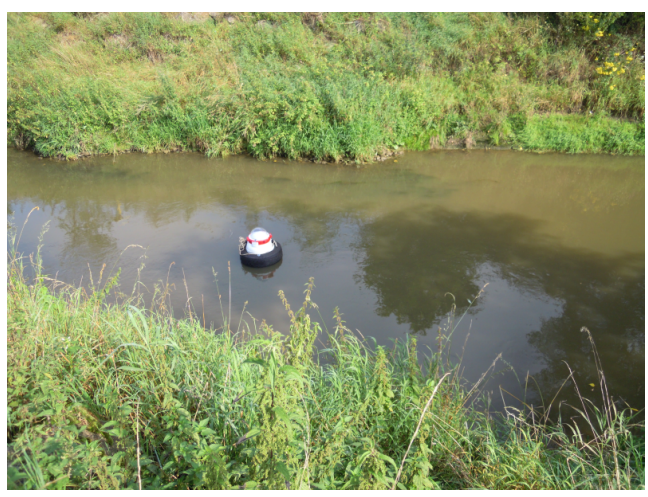

(b)

Figure 1: Photograph of the GPS buoy (a) outside the water and (b) drifting in the Alzette river stream.

(2012) this kind of smoother can be compared to vari- 277 ational assimilation techniques.

The implementation used in this study is based on ${ }^{279}$ the Sequential Importance Sampling (SIS) method. In ${ }^{280}$ our study, each particle consists of a possible state of ${ }^{281}$ the variables, namely the WSE simulated over time and ${ }^{282}$ space using one hydrodynamic model. Each hydro- ${ }^{283}$ dynamic model is based on one realization of the bathy- ${ }^{284}$ metry. In other words, one particle correspond to the ${ }^{285}$ WSE (distributed over space and time) simulated by one ${ }^{286}$ hydrodynamic model using one bathymetry realization. ${ }^{287}$ Section 2.3 explains how the ensemble of bathymetry ${ }^{288}$ realization is generated. The number of state variables ${ }^{289}$ for a given particle corresponds to the number of ele- ${ }^{290}$ ments in the geometry of the model domain multiplied ${ }^{29}$ by the number of time steps. In this study, we make use of a one-dimensional hydrodynamic model (see section 2.3.1). In such a model, the geometry is defined by cross sections perpendicular to the main flow direction. For simplifying the riverbed representation and facilitating the data assimilation process, it is assumed here that the targeted riverbed bathymetry is of rectangular shape (see Figure 2) with a known river width (RW). The riverbed is thus characterized by only one parameter, the river depth (RD). Of course, real riverbeds 298 are rarely of rectangular shape. However, we believe that this simplification is sensible for this study as it allows validating the new concept and, in the same time, 299 does not prevent the model from generating realistic res- 300 ults. It is also worth mentioning that the same assump- 301 tion has been made in similar proof-of-concept studies 302 (e.g. Durand et al., 2008; Yoon et al., 2012). Finally, the 303 method introduced in this study can be extended to more 304 complex geometries.
In a PF, the filtering density is approximated by a discrete distribution, whose support is the set of particles. The probability mass assigned to each particle is proportional to that particle's weight, which, in turn, is proportional to the likelihood of the observation at the assimilation time step (Fearnhead, 2002). The particles are sampled directly from the state-space to represent the posterior probability, and a local weight is computed for each particle at each cross section and at each time step, according to the information contained in the observations: a local weight, $\mathrm{w}^{i, j, k}$, is assigned to an observed cross section $\mathrm{j}$ for a particle $\mathrm{i}$, at an observation time step k. Note that the weighting procedure can be adapted to any kind of distribution function. When assimilating data stemming from the GPS buoy, a Gaussian likelihood was used, assuming the mean of recorded WSE to represent the mean of a normal distribution whose shape is defined by a pre-defined value of standard deviation. A local weight, $\mathrm{w}^{i, j, k}$, is therefore computed for a WSE $\mathrm{x}^{i, j, k}$ simulated by particle $i$ at cross section $j$ and time step $k$, for which an observation is available:

$$
w^{i, j, k}=\frac{1}{\sigma \sqrt{2 \pi}} e^{\frac{-\left(x^{i, j, k}-\mu^{i, j}\right)^{2}}{2\left(\sigma^{i, j}\right)^{2}}}
$$

In Eq. 1, $x$ is the matrix of the state variables (WSE at cross section $j$ and time step $k$ simulated by particle $i$ ), $\mu$ is the observation mean and $\sigma$ is the standard deviation associated with the observations. The matrix of weights contains all local weights, $\mathrm{w}^{i, j, k}$. Subsequently, one global likelihood is computed for each particle and each cross section by applying the joint probability the- 
ory for independent variables:

$$
\omega^{i, j}=\prod_{k=1}^{N_{o}} w^{i, j, k}
$$

where $\mathrm{N}_{o}$ is the number of observation time steps. The resulting global weights are then normalized using 351 Eq. 3.

$$
W^{i, j}=\frac{\omega^{i, j}}{\sum_{i=1}^{N_{p}} \omega^{i, j}}
$$

In Eq. 3, $\mathrm{N}_{p}$ is the number of particles. Next, the global weights allow for the computation of an expectation of the updated WSE:

$$
E\left(x^{i, j, k}\right)=x_{e x p}^{j, k}=\sum_{i=1}^{N_{p}} W^{i, j} x^{i, j, k}
$$

To update the riverbed bathymetry, we propose to cor- 364 rect each river bathymetry realization by shifting the 365 corresponding riverbed bottom elevation using a dis- 366 tance equal to the time-averaged deviation between the ${ }^{367}$ WSE simulated by this particle and the expected WSE: ${ }^{368}$

$$
U p B O E_{i, j}=B O E_{i, j}+\sum_{k=1}^{N_{o}} \frac{x^{i, j, k}-x_{e x p}^{j, k}}{N_{o}}
$$

In Eq. $5 B O E_{i, j}$ and $U p B O E_{i, j}$ represent respectively the first guess and the updated bathymetry (for particle $i$ at model cross section $j$ ) as shown in Figure 2. It is worth noting that each cross section bathymetry is up- 374 dated with an independent $\delta^{i, j}=\sum_{k=1}^{N_{o}} \frac{x^{i, j, k}-x_{e x p}^{j, k}}{N_{o}}$. 375

Subsequently, new model simulations are performed 376 using the updated bathymetry realizations, before a new ${ }^{377}$ assimilation process is carried out. This bathymetry up- ${ }^{378}$ date cycle is then repeated until a convergence of bathy- ${ }^{379}$ metry realizations is obtained (i.e. until a predefined ${ }^{380}$ tolerance criterion is satisfied).

Figure 2 presents the flowchart of the assimilation 382 scheme.

\subsection{Designing the synthetic experiment}

In this part of the paper we propose a synthetic ex- 386 periment in order to evaluate the value of GPS-derived 387 WSE measurements for retrieving riverbed bathymetry. 388 This experiment makes use of a calibrated hydro- 389 dynamic model. The next paragraph (see 2.3.1) intro- 390 duces this model and its calibration. Paragraph 2.3.2 391 explains how the synthetic observations have been gen- 392 erated using this model. Finally, paragraph 2.3 .3 pro- 393 poses a method for generating the ensemble of model 394 simulations.

\subsubsection{The hydrodynamic model}

The set up and calibration of the hydrodynamic model is described in Hostache et al. (2009). This model has been developed using the freely available Hec-RAS software (USACE, 2002). Hec-RAS performs unsteady simulations by solving the 1Dimensional de Saint-Venant equation using an implicit finite difference approximation. Hostache et al. (2009) calibrated two Manning friction coefficients: one for the river channel $\left(n_{c}=.047\right)$ and one for the floodplain $\left(n_{f l p}=.182\right)$. During the calibration process, MonteCarlo simulations within ranges of plausible Manning parameters were carried out. The upstream boundary condition was the observed discharge hydrograph. The parameter set providing the best performance with respect to both observed downstream discharge hydrographs and remote sensing-derived WSE distributed across the floodplain was selected as optimal (see Hostache et al., 2009, for more details). It has to be noted here that in spite of the necessity to carry out the experiment in a realistic set up, the calibration of the hydrodynamic model is not the main issue of the study. Indeed, the objective is to retrieve an effective bathymetry that allows the model to yield correct predictions of flood wave propagation in terms of discharge and water surface elevation. Therefore, slightly different values of friction would most likely result in slightly different values of the retrieved effective bathymetry.

\subsubsection{The synthetic observations}

The method that was adopted for generating synthetic observations aims at creating a synthetic dataset that is representative of a dataset that could be obtained from the GPS buoy. To do so, we propose a two-step approach, namely (i) to generate a so-called synthetic truth by a forward run of the calibrated hydrodynamic model, (ii) to perturb the so-called truth in order to generate synthetic observations with controlled errors.

In the experiment, we assume that for several days a buoy is launched at the upstream end of the river reach. This is done every day at the same time. The buoy is left drifting freely in the river and is then recovered when it reaches the downstream end. This means that many free drifting cycles (one per day) of measurements are obtained from such an experiment.

The one-dimensional hydrodynamic model (see 2.3.1) provides as output the cross section-averaged flow velocity and the WSE at each cross section of the model for every time step of the simulation. We obtained the synthetic truth from a forward run of the calibrated hydraulic model. In our scenario, a buoy is 


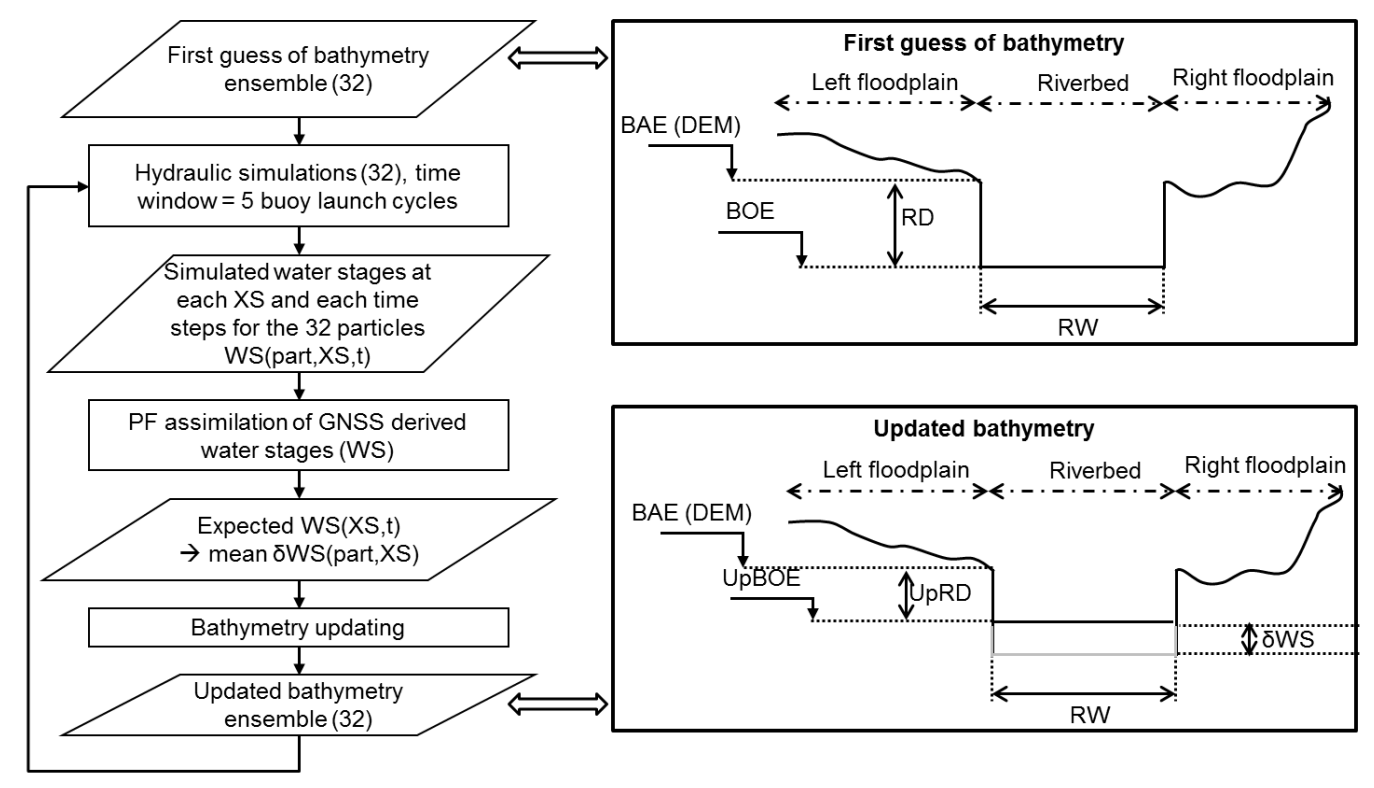

Figure 2: Flowchart of the assimilation method.

launched at the upstream boundary of the river. As- 424 suming that the buoy velocity equals the cross section- 425 averaged flow velocity provided by the model, it is pos- 426 sible to predict the buoy position at every step, by se- ${ }_{427}$ quentially multiplying the velocity value by the time 428 step duration. By repeating this computation for each ${ }_{429}$ time step, it is then possible to estimate the position of ${ }_{430}$ the synthetic buoy. As the model also provides WSE, 431 the synthetic truth consists of the buoy location, namely ${ }_{432}$ the model cross section on which it is located, and the ${ }_{433}$ corresponding simulated WSE.

Finally, synthetic WSE observations are derived by 435 adding to the synthetic truth a normally distributed ${ }_{436}$ noise, whose mean and standard deviation are set to val- ${ }_{437}$ ues that are representative of the data that are expected ${ }_{438}$ to be obtained using the actual GPS. It is worth noting ${ }_{439}$ that, at each time step, only one value of WSE at a given ${ }_{440}$ position is provided by the synthetic GPS buoy.

\subsubsection{The particle ensemble set up}

Our experiment consists of assimilating synthetic ${ }_{444}$ WSE observations into an ensemble of hydraulic mo- ${ }_{445}$ dels, whose upstream boundary condition is a flow hydrograph obtained by the means of continuous in situ 446 WSE measurements and a rating curve.

The truth and the ensemble of model predictions were ${ }_{448}$ generated separately: they share the same model struc- ${ }_{449}$ ture, parameters, forcings and initial conditions, how- 450 ever the geometry components differ. In fact, to cre- 451 ate the so-called truth, a hydraulic model run has been performed using the observed geometry composed of a floodplain Lidar DEM and a ground surveyed riverbed bathymetry (see section 2.3.2). For the bathymetry retrieval exercise, on the other hand, we removed the bathymetry information and we assumed the riverbed to be of rectangular shape (see Figure 2). To create the ensemble of model predictions, we randomly generate 32 possible realizations, representing a first guess of the bathymetry. To do so, the unknown riverbed shape is assumed to be of rectangular type, with a given river depth (RD) and a given river width (RW) (see Figure 2). It is assumed here that the bank elevation (BAE, see Figure 2) and the floodplain geometry are determined using an available digital elevation model (e.g. the globally available SRTM DEM with a spatial resolution of $90 \mathrm{~m}$ ). We also suppose that the river width RW can be obtained from optical satellite data or any other source of information. According to these two assumptions, the river bathymetry is defined by the river depth RD (Figure 2), with the river bottom elevation BOE being obtained by subtracting RD from BAE.

For each cross section of the hydrodynamic model, random values of RD are generated in order to obtain a first guess of the bathymetry. To maintain a certain hydraulic consistency of the randomly generated river depth of each particle, we suggest the following twostep approach: 
1. random generation of 32 plausible values of RD ${ }_{503}$ (one for each particles), that are subtracted from BAE values along the river reach to obtain the bed 504 level for each particle,

2. random perturbation at the cross section level (er- 506 ror with uniform distribution centered on 0 and ${ }^{507}$ with a range equal to $25 \%$ of the randomly gen- 508 erated RD value for the specific particle).

This two-step approach ensures a good computational 511 stability of the hydrodynamic model, as non-plausible 512 RD values could lead to more time-consuming and fail- 513 ing computations. It has to be noted here that we expect 514 the number of bathymetry realizations not to be of ma- 515 jor importance. The number of realizations does have 516 some influence on the time it takes for the assimilation 517 algorithm to converge. However, our experiments show 518 that the final bathymetry estimate is not significantly af- 519 fected by the number of particles. As a matter of fact, 520 the number of realizations has been set to 32 as this 521 number was considered a good compromise between ${ }_{522}$ computational efficiency and convergence capability. In ${ }_{523}$ our opinion, the key point for efficiently retrieving the ${ }_{524}$ bathymetry is to ensure that the spread of the realiza- 525 tions encompasses all the values that could a priori be ${ }_{526}$ expected for the real bathymetry. To evaluate the gen- ${ }_{527}$ erated ensemble, we computed two verification meas- ${ }_{528}$ ures proposed by De Lannoy et al. (2006), namely the ${ }_{529}$ ratio between the averaged ensemble skill and the aver- 530 aged ensemble spread (called $V M_{1}$ hereafter) and the ratio between the average squared ensemble skill and the averaged root mean squared error computed between simulation and observation (called $V M_{2}$ hereafter). According to De Lannoy et al. (2006), $V M_{1}$ might be close to one to guaranty that the ensemble spread is of the order of magnitude of the model deviation to observation. A value of $V M_{1}$ higher than 1 means that the ensemble spread is too small whereas a value of $V M_{1}$ lower than one means that the ensemble spread could be further re- ${ }^{536}$ duced. According to De Lannoy et al. (2006), with 32 537 particles, $V M_{2}$ might be equal to 0.72 for the observa- ${ }_{538}$ tion and the ensemble to be statistically undistinguish- ${ }_{539}$ able.

At this stage of the methodology, a first guess of the ${ }_{541}$ geometry has been defined via a set of 32 realizations of ${ }_{542}$ the river bathymetry. Each particle corresponds to a hy- ${ }_{543}$ drodynamic model making use of one bathymetry reali- ${ }_{544}$ zation. It is worth mentioning that for a given particle, 545 the RD value is different at each cross-section. After ${ }_{546}$ the first guess of the bathymetry has been generated, the ${ }_{547}$ assimilation algorithm as defined in section 2.2 is ap- ${ }_{548}$ plied.

\section{Study area and available data}

The area of interest is located in the Grand Duchy of Luxembourg (see Figure 3).

The basin area is about $356 \mathrm{~km}^{2}$ at the stream gauge located in Pfaffenthal where WSE is recorded every $15 \mathrm{~min}$. The corresponding discharge hydrograph has been estimated using the rating curve of this hydrometric station. It constitutes the upstream boundary condition of the hydrodynamic model, which simulates the $19 \mathrm{~km}$ reach of the Alzette River between the hydrometric stations at Pfaffenthal and Mersch (Hostache et al., 2009). The river reach is described by 158 groundsurveyed channel cross sections whereas the floodplain topography has been extracted from a Lidar DEM of $2 \mathrm{~m}$ pixel spacing and $15 \mathrm{~cm}$ vertical accuracy (see Hostache et al., 2009, for more details). Moreover, aerial photographs with $50 \mathrm{~cm}$ spatial resolution were available for the area of interest and used to determine river width.

The synthetic experiment is grounded on a real storm event starting on January 22012 and ending on January 6 2012. During these five days there were high variations of discharge without overbank flooding. These characteristics of the event are rather important, as they imply that the buoy would have kept drifting inside the riverbed during the experiment and would have provided WSE observations associated with different flow conditions.

\section{Results and discussion}

This section first presents and discusses the results of "dry" tests (i.e.: carried out outside the water) of the GPS buoy. Next it focuses on the data assimilation experiment and discusses its outcome.

\subsection{Evaluating the vertical accuracy of the GPS buoy}

The "dry" tests that have been carried out provide some insights into the accuracy levels that can be achieved when deploying the buoy inside a river. The road and the parking lot around the research institute (Belvaux, Luxembourg) offer an appropriate test site for the system. With the "canyon" of surrounding buildings and their impact on the GPS signal, the study site shares some similarities with a river channel where double bounce effects caused by trees and the river banks cause also perturbations. Moreover, a loss of signal is possible due to the surrounding trees, banks or buildings, that also mask out part of the sky, thereby reducing the number of available GPS satellites. For this study site, the 

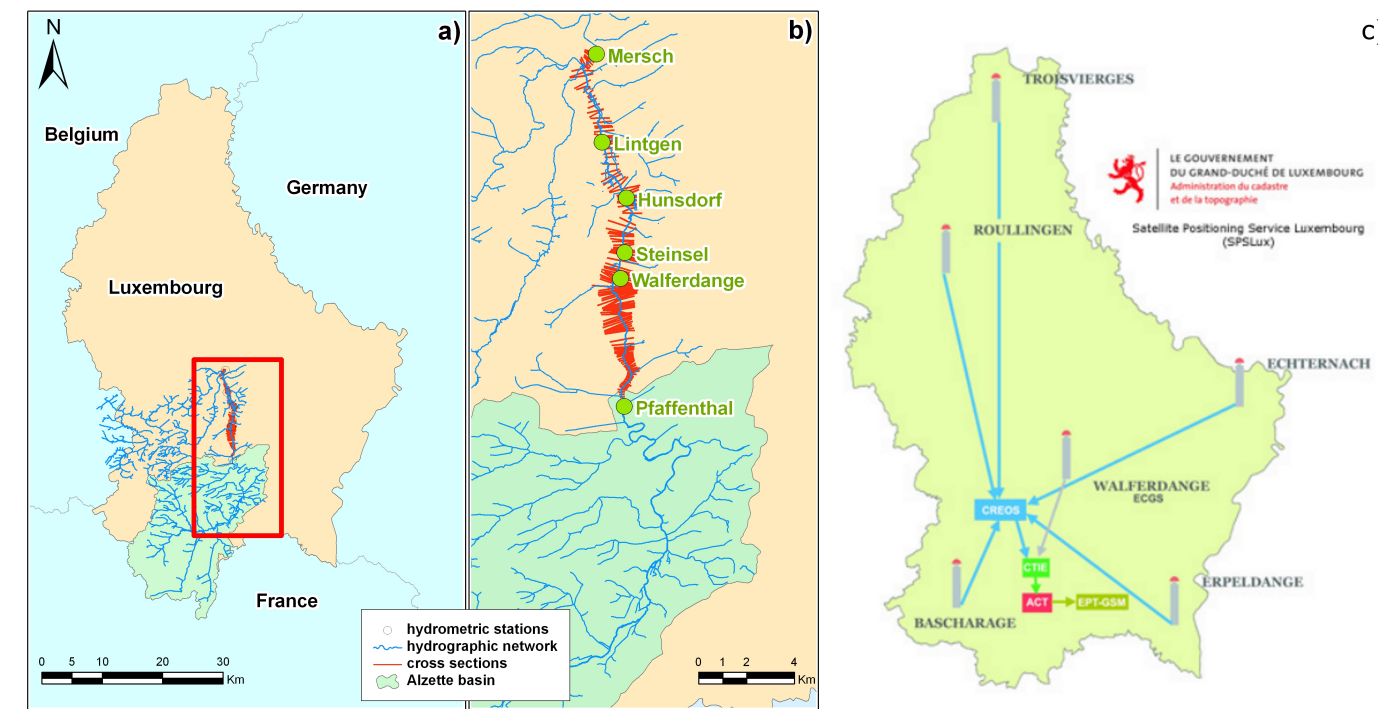

Figure 3: Study site in the Alzette River basin showing: (a) the drainage area down to Pfaffenthal and the $19 \mathrm{~km}$ river reach whose geometry is represented by the cross sections, (b) the hydrometric stations along the river, c) the SPSLux refence GPS station map (source http://www.act.public.lu).

closest SPSLUX reference station is located in Baschar- 579 age at a distance of $16 \mathrm{~km}$ (see Figure 3c).

To carry out the experiment in "dry" conditions, the 581 first step was to define a set of reference points with 582 accurately-known geographic coordinates and altitudes. ${ }_{583}$ To do so, a theodolite was used, enabling the positioning 584 with an associated planimetric and altimetric accuracy of less than $1 \mathrm{~cm} .60$ reference points have been marked on the ground and accurately located on the site. Next, 585 by moving the GPS buoy along the pre-defined path and using a stopwatch as time recorder, it was possible to 586 estimate the accuracy of the positioning. Each time the ${ }_{587}$ GPS receiver passed over a mark on the ground, the re- 588 lative time from the beginning of the test was recorded 589 using the stopwatch. This relative time was converted 590 into absolute time using the GPS time at the beginning 591 of each test. The known position of the marks was then 592 compared with the position measured by the GPS at the ${ }_{593}$ time recorded by the stopwatch.

The results of this test showed that the elevation in- 595 formation provided by the system may be biased if spe- 596 cific time steps are considered. However, over four test 597 campaigns spread over two days and at various day- 598 times, it appeared that the mean altimetric error equaled 599 $0 \mathrm{~cm}$ and its standard deviation $30 \mathrm{~cm}$. Figure 4 presents 600 the GPS altimetric measurement errors recorded during 601 the experiment. These values were used as a basis for 602 defining the observational errors in the synthetic exper- 603 iment.
It is worth mentioning here that other GNSS could be used in addition to GPS, like for instance Glonass and the upcoming Galileo, in order to increase the GNSS measurement accuracy and to avoid signal losses especially when the number of visible satellites becomes critically low.

\subsection{Generating synthetic observation}

To create synthetic observations we followed the procedure presented in paragraph 2.3.2. To do so, we performed a forward run of the calibrated hydraulic model using the measured geometry data. The upstream boundary condition used for this model run was the discharge hydrograph recorded by the Pfaffenthal stream gauge. During this model run (between January 2 and 6 2012), with a simulation time step of one minute, the simulated WSE and the cross-section-averaged flow velocities at all the cross sections have been stored. Assuming that a buoy is launched every day at 7:00 AM and drifts freely at the cross-section-averaged flow velocity, the results of the hydrodynamic simulation allow calculating the position of the buoy and the corresponding WSE at each time step (see section 2.3.2). The position and the elevation of the buoy represent the so-called "truth". Next, the "truth" has been perturbed using a normally distributed noise with a mean of $0 \mathrm{~m}$ and a standard deviation of $30 \mathrm{~cm}$. 


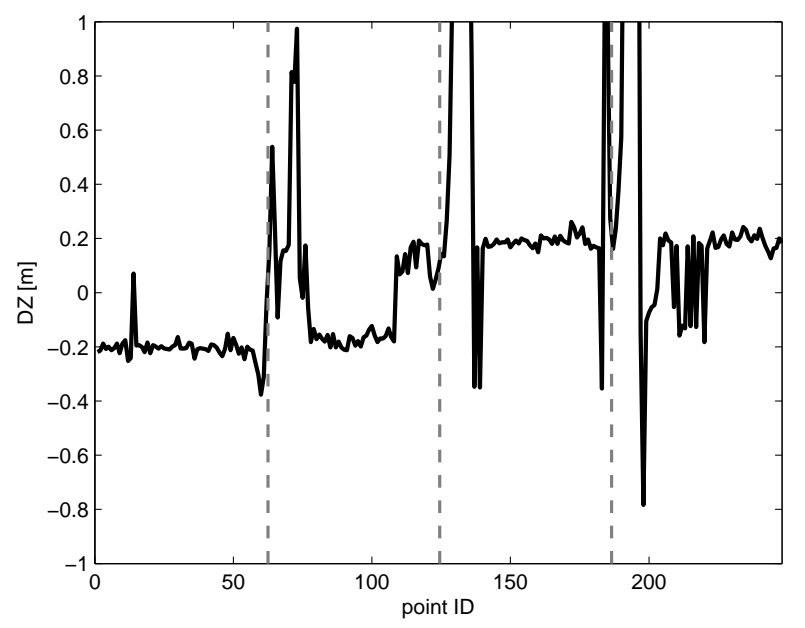

Figure 4: Summary of measurement error during the GPS dry condition test. The black line represent the measurement error whereas the gray dotted vertical lines identify the four field campaigns.

\subsection{Retrieving bathymetry via assimilation of synthetic 635 observations}

The aim of the synthetic experiment is to evaluate the ${ }^{637}$ potential added value of WSE measurements provided 638 by the developed GPS buoy for bathymetry retrievals. ${ }^{639}$ As introduced in section 2.2, the assimilation proced- 640 ure is carried out iteratively using the full set of WSE ${ }^{641}$ observations.

As proposed in paragraph 2.3.3, to create the en- 643 semble of model predictions 32 realizations of the 644 bathymetry were generated. Bank elevation (BAE, see 645 Figure 2) and floodplain geometry were extracted for 646 each cross-section from the available Lidar DEM. The 647 river widths RW were determined by digitizing the river 648 banks using the aerial photographs (see section 3). The 649 river bathymetry is then created by randomly generating 650 RD values following the two-step approach proposed in 651 section 2.2:

1. 32 plausible values of RD were drawn from a uniform distribution ranging between 0 and $20 \mathrm{~m}$ (one for each particle) and subtracted from the BAE value along the river reach to obtain the bed level for each particle,

2. a random noise was added to each cross-section riverbed (error with uniform distribution centered on 0 and with a range equal to $25 \%$ of the randomly generated RD value for the specific particle).

The first guess of the bathymetry is presented in Figure 5a. In this figure, each light gray line corres- 665 ponds to one particle of the ensemble, whereas the bold black and grey lines represent the observed bathymetry and the ensemble mean, respectively. It is worth specifying that what we call observed bathymetry is actually not the lowest point of the riverbed extracted from the ground surveyed cross sections. Instead, considering that the ground-surveyed channel cross sections do not have a rectangular shape, we computed an equivalent rectangle-shaped bathymetry, having hydraulic properties equivalent those of the observed one. In other words, from each real cross-section we computed the "observed" RD so that the corresponding rectangle-shaped cross-section has, under the assumption of steady flow conditions, a rating curve (discharge/WSE relationship) as close as possible to that of the true riverbed cross-section. This computation has been carried out iteratively, based on the optimization of the Manning-Strickler formula. As a matter of fact, the so-called observed bathymetry corresponds to the rectangular-shaped equivalent of the true bathymetry.

As proposed in section 2.3.3, to evaluate the generated ensemble, we computed two verification measures proposed by De Lannoy et al. (2006). In our study, computed on WSE, $V M_{1}$ is equal to 0.62 which means that the ensemble spread could be further reduced but can however be correctly used. Furthermore, $V M_{2}$ is equal to 0.61 , which means that the ensemble and the observation are statically distinguishable, but similar. As a matter of fact, the two verification measures indicate that the 32 generated particles can be used correctly in an assimilation framework. 
Figure $5 \mathrm{~b}, \mathrm{c}$ and $\mathrm{d}$ show, respectively, the results of 718 the assimilation after iterations 1, 3 and 10. In Fi- 719 gure 5a, it is worth noting that the spread of the en- 720 semble first guess is rather large and that the ensemble 721 mean is distant from the bathymetry observation. After 722 the first iteration, Figure 5b shows that the spread of the 723 particles is significantly reduced. After three iterations, 724 the spread of the particles is more reduced (Figure 5c) 725 and, after ten iterations (Figure 5d), the ensemble fully 726 converges, i.e. all the particles overlap. In addition, Fi- 727 gure 6 presents the water surface elevation lines (along 728 the river reach) simulated by the model at a time step 729 close to the flood peak, for various assimilation itera- 730 tions. This figure demonstrates that the simulated water 731 levels quickly converge toward the so-called synthetic 732 truth.

Figure 7 shows two performance criteria of the up- 734 dated bathymetry and one performance criterion of the 735 simulated WSE computed after each iteration of the assimilation algorithm. In Figure 7, the black line represents the spread of the bathymetry ensemble. This line 736 shows that the spread is almost reduced to zero after 8 iterations. In the same figure, the light and middle grey 737 lines correspond respectively to the root mean squared 738 error (RMSE) and the mean error (ME) between the 739 average of the bathymetry ensemble and the observed 740 bathymetry. When analyzing the behaviour of the en- 741 semble spread, the RMSE and the ME are quite similar, 742 in the sense that they reach a plateau after several itera- 743 tions. The lowest RMSE that is reached after several it- 744 erations equals $36 \mathrm{~cm}$, whereas the lowest obtained ME 745 is close to $0 \mathrm{~cm}$. These results are encouraging, as they 746 demonstrate that the observed and the retrieved bathy- 747 metries are in good agreement. An iterative repetition 748 of the assimilation technique allows for an efficient re- 749 trieval of the bathymetry.

When having a closer look at Figure 5d, one can no- 751 tice that the general trend of the observed bathymetry is 752 well reproduced, despite the local topography not being 753 described in all details. This indicates that the method 754 enables the computation of the main characteristics of 755 the bathymetry, but has its limitations for describing 756 bathymetry changes at small scale. The retrieved bathy- 757 metry indeed appears as a smoothened estimate of the 758 true bathymetry. To understand the origin of this ef- 759 fect, it is important to consider how the bathymetry in- 760 fluences WSE. Only bathymetry features having a sig- 761 nificant effect on the WSE have a chance of being re- 762 trieved using the method. It can be argued that some 763 of the small scale bathymetry features only have a lim- 764 ited effect on the WSE. Consequently, given the WSE 765 measurement uncertainty ( $30 \mathrm{~cm}$ in this study), these 766 features are not detectable with the proposed method.

In addition, Figure 7 shows the RMSE calculated between the mean of the simulated WSE ensemble and the truth. It is worth noting that after four iterations, the RMSE computed on WSE reaches a plateau at a value equalling $27 \mathrm{~cm}$. This result is also encouraging as it shows that the WSE is correctly simulated despite the small scale bathymetry not being retrieved in all its details. Furthermore, this shows that the hypothesis of a rectangular shaped riverbed is acceptable as in spite of this simplification the model reaches a satisfying level of accuracy in terms of simulated water levels. Moreover, this $27 \mathrm{~cm}$ value must be mirrored with the error of $30 \mathrm{~cm}$ imposed to the synthetic GPS measurements. This demonstrates the reliability of the assimilation technique as the error of the simulated WSE is lower than the error of the synthetic observations.

\section{Conclusions}

The study presented in this paper focused on the potential benefits deriving from assimilating WSE observations provided by a drifting GNSS-equipped buoy into a hydrodynamic model for effective bathymetry retrievals.

A GPS buoy has been designed for measuring WSE. To reach a satisfactory accuracy level of WSE measurements, this buoy includes an integrated dualfrequency GPS, namely the Hemisphere A221 ${ }^{T M}$ Smart Antenna, used in differential mode. By testing this GPS in "dry" conditions we were able to estimate an elevation measurement accuracy of $30 \mathrm{~cm}$. For the assimilation exercise, in order to keep control on the model and measurement errors we carried out synthetic experiments. This allowed us to analyze, in a controlled environment, the added-value the GNSS-derived data sets may provide to hydraulic modeling and bathymetry retrievals. In this paper, we only made use of GPS, but it is worth noting that the extension to other GNSS, such as the upcoming Galileo, is feasible and may improve the GPS measurement accuracy.

The assimilation algorithm that was used is based on the Particle Filter, following the work of Giustarini et al. (2011). The proposed variant of the PF, termed in this paper particle smoother, is based on a global weighting procedure: a single particle contains, as state matrix, WSE at all cross sections and all time steps. The likelihood of each particle is derived from its ability to correctly predict WSE at the buoy's locations. Next, these likelihoods are used to estimate an expectation of the 


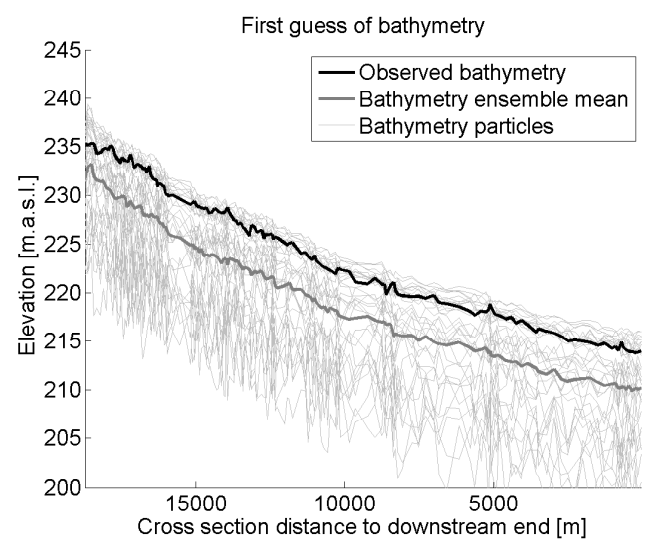

(a)

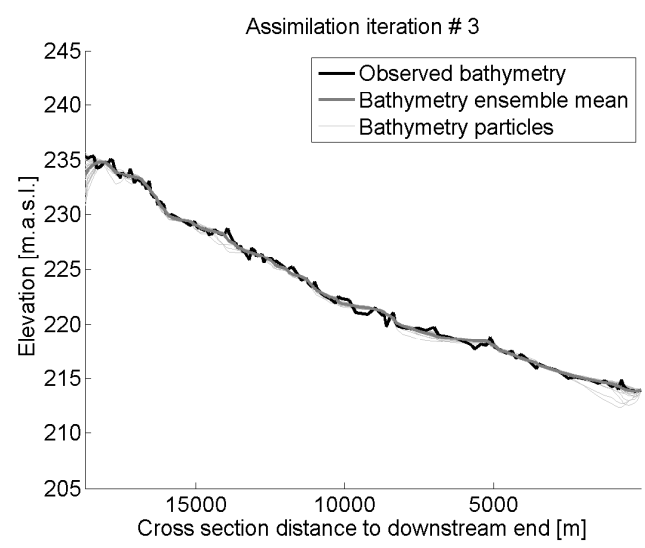

(c)

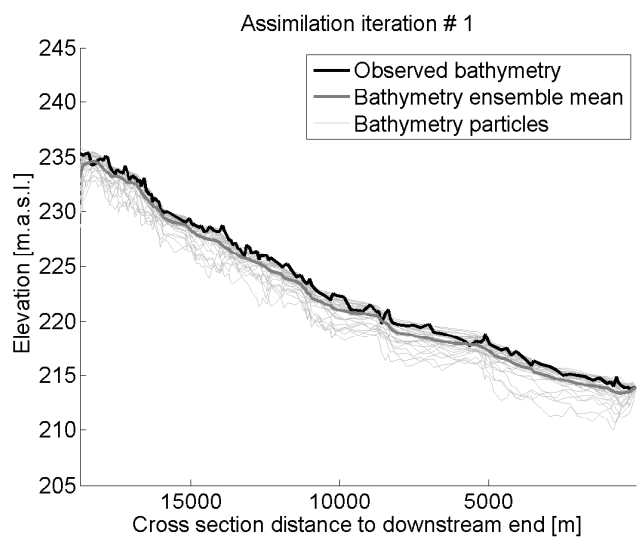

(b)

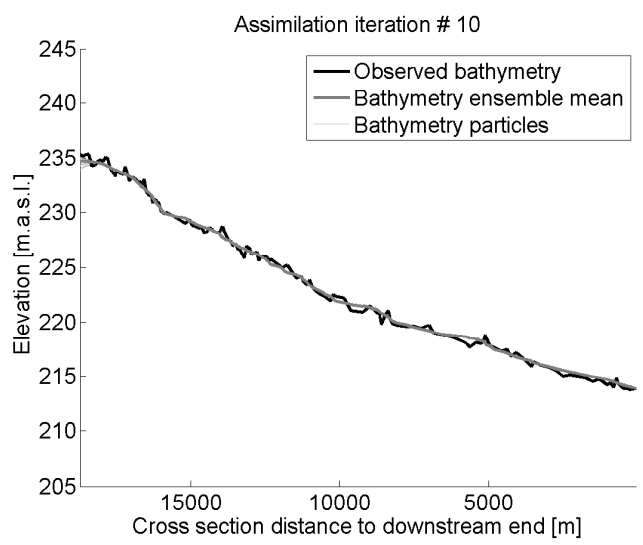

(d)

Figure 5: Bathymetry retrievals: first guess (a), and updated bathymetry after assimilation iterations 1 (b), 3 (c) and 10 (d). 


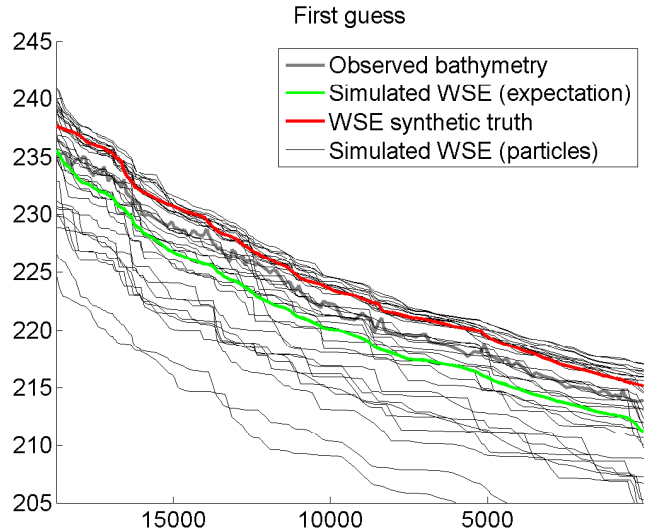

(a)

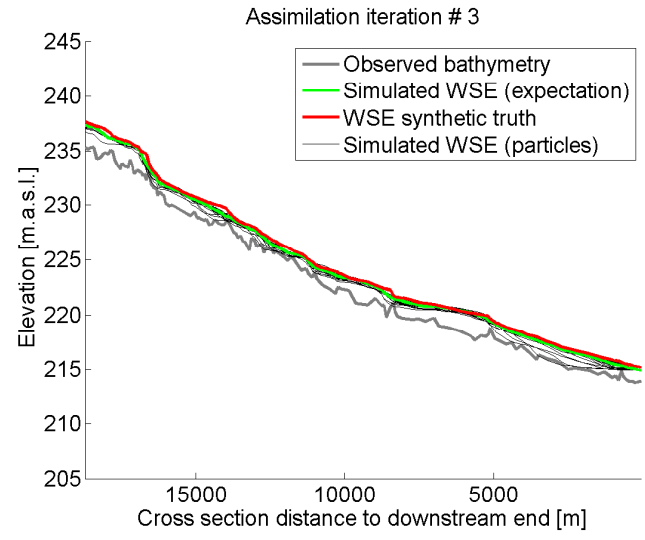

(c)

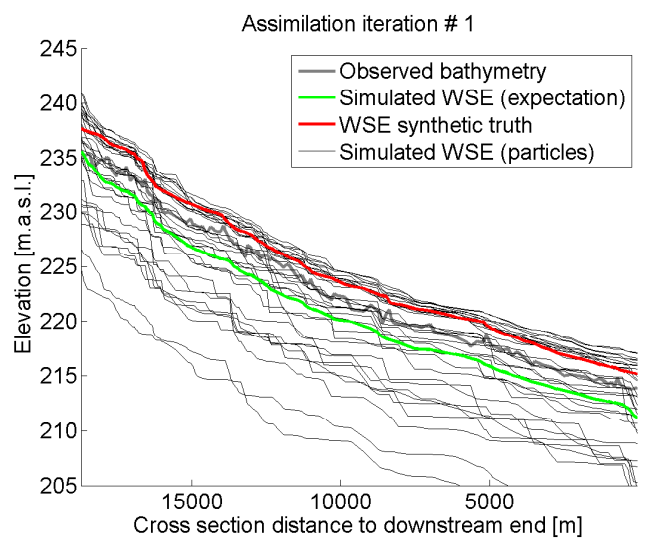

(b)

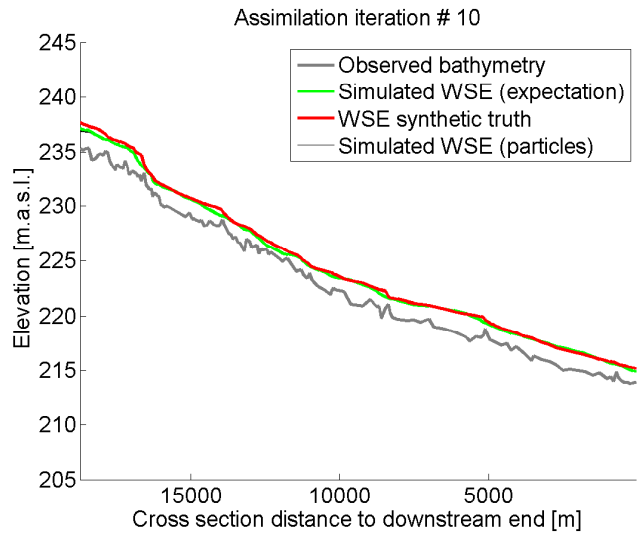

(d)

Figure 6: Simulated water surface elevation profiles (close to flood peak) after various assimilation iteration : open loop (a), and after assimilation iterations 1 (b), 3 (c) and 10 (d). 


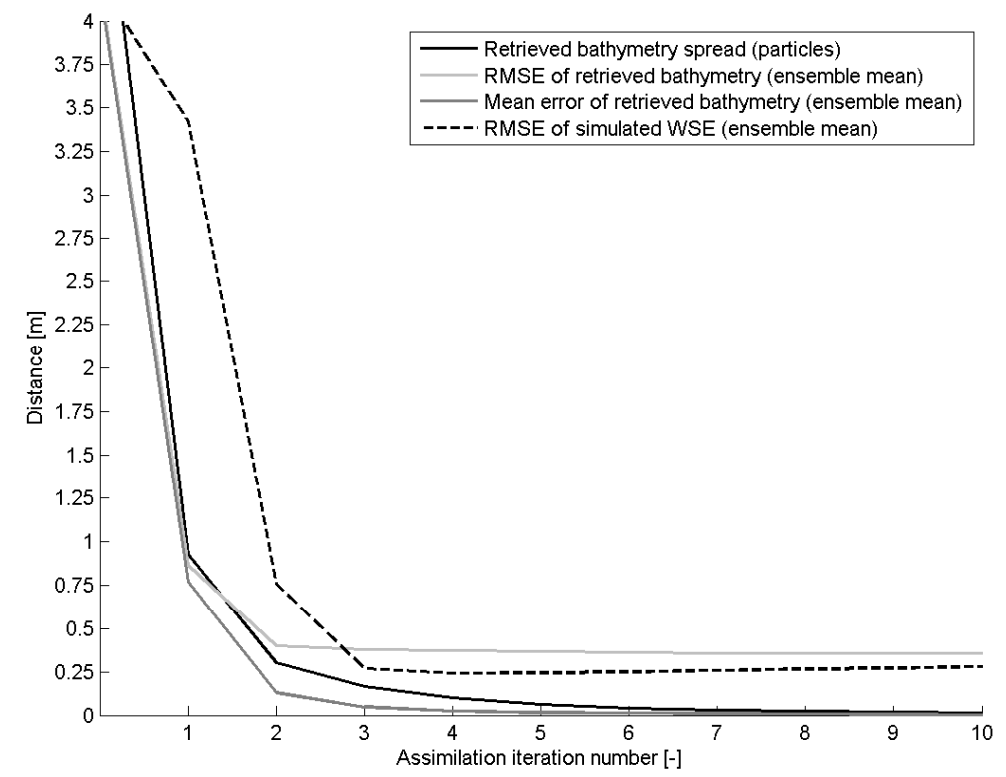

Figure 7: Bathymetry retrieval performance

WSE, that is later used to update the rectangular-shaped 793 bathymetry associated with each particle.

In the assimilation scenario proposed in this study, one buoy is launched once a day during five subsequent days, implying that at a given time a single buoy is drifting along the channel. The assimilation is performed iteratively, in order to get gradually closer to the true bathymetry. The results show that the method enables the retrieval of the bathymetry with an accuracy of $36 \mathrm{~cm}$. This result is promising and slightly better than the $56 \mathrm{~cm}$ and $52 \mathrm{~cm}$ obtained in similar studies by Durand et al. (2008) and Yoon et al. (2012). The general trend of the observed bathymetry is well reproduced while some of the small scale bathymetry features were missed out. The simulated WSEs are also in good agreement with the synthetic truth as the computed root mean squared error is equal to $27 \mathrm{~cm}$.

The next step will be to carry out similar experiments with actual measurements. One further development of 811 the assimilation and updating technique will be to ana- ${ }^{812}$ lyze the added value of considering more realistic crosssection shapes, like a trapezoidal shaped cross-section. 815 Moreover, one key issue of such techniques will be 816 the retrieval of Manning friction coefficients in addi- ${ }^{817}$ tion to bathymetry, as already proposed by Durand et al. ${ }_{819}^{818}$ (2008).

\section{Acknowledgements}

This study was part of the FLOODTRACKER project, financed by the National Research Fund (FNR) of the Grand Duchy of Luxembourg in the framework of the CORE research program (Contract no. C09/SR/19). Giovanni Corato's contribution was supported by the National Research Fund of Luxembourg through the FLOODMOIST project (SR/02/152).

We would like to thank several people with whom we discussed these ideas over the past years. We would like to mention in particular Laurent Pfister (with CRPGabriel Lippmann). We would like also to thank several people for sharing their expertise in GNSS with us, in particular Willy Croi, Arnaud Vacelet, Joan Fort (Luxspace S.A.), Tonie van Dam (University of Luxembourg) and Bernard Reisch (Administration du Cadastre et de la Topographie, Luxembourg).

\section{References}

Alsdorf, D., Rodriguez, E., Lettenmaier, D., 2007. Measuring surface water from space. Rev. Geophys. 45.

Andreadis, K. M., Clark, E. A., Lettenmaier, D. P., Alsdorf, D. E., 2007. Prospects for river discharge and depth estimation through assimilation of swath-altimetry into a raster-based hydrodynamics model. Geophys. Res. Lett. 34, 5.

Apel, H., Hung, N., Thoss, H., Schne, T., 2012. Gps buoys for stage monitoring of large rivers. J. Hydrol. 412-413, 182-192.

Biancamaria, S., Durand, M., Andreadis, K., Bates, P., Boone, A., Mognard, N., Rodriguez, E., Alsdorf, D., Lettenmaier, D., Clark, 
E., 2011. Assimilation of virtual wide swath altimetry to improve 88 Arctic river modeling. Remote Sens. Environ. 115, 373-381.

Bisnath, S., Wells, D., Howden, S., Dodd, D., Wiesenburg, D., 2003. 88 Development of an operational RTK GPS-equipped buoy for water 889 level recovery. In: Proceedings of ION GPS/GNSS 2003. Portland, 890 OR, 9-12 September 2003.

Burgers, G., Leeuwen, P. J. V., Evensen, G., 1998. Analysis scheme in 892 the ensemble Kalman filter. Mon. Weather Rev. 126, 1719-1724. 893

De Lannoy, G. J. M., Houser, P. R., Pauwels, V. R. N., , Verhoest, 894 N. E. C., 2006. Assessment of model uncertainty for soil moisture 895 through ensemble verification. J. Geophys. Res. 111 (D10101). 896

Dunne, S., Entekhabi, D., 2005. An ensemble-based reanalysis ap- 897 proach to land data assimilation. Water Resour. Res. 41.

Durand, M., Andreadis, K. M., Alsdorf, D. E., Lettenmaier, D. P., 899 Moller, D., Wilson, M., 2008. Estimation of bathymetric depth 900 and slope from data assimilation of swath altimetry into a hydro- 901 dynamic model. Geophys. Res. Lett. 35 (20), L20401.

Evensen, G., 1994. Sequential data assimilation with a nonlinear quasigeostrophic model using Monte Carlo methods to forecast er- 904 ror statistics. J. Geophys. Res. 99 (C5), 10143-10162.

Fearnhead, P., 2002. Markov chain Monte Carlo, sufficient statistics, 906 and particle filters. J.Comput. Graph. Stat. 11, 848-862.

Giustarini, L., Matgen, P. Hostache, R., Montanari, M., Plaza, D. Pauwels, V. R. N., De Lannoy, G. J. M., De Keyser, R., Pfister, 909 L. Hoffmann, L., Savenije, H. H. G., 2011. Assimilating SAR- 910 derived water level data into a hydraulic model: a case study. Hy- 911 drol. Earth Syst. Sci. 15, 2349-2365.

Holtschlag, D. J., Aichele, S., 2001. Visualization of drifting buoy 913 deployments on St. Cr River near public water intakes. Tech. rep., 914 U.S. Geological Survey Open File Report 01-17.

Hong, J., Backa, K., Park, U., Lee, D., Cha, S., 2008. Determination 916 of ocean datum using GPS buoy observation data. In: The Interna- 917 tional Archives of the Photogrammetry, Remote Sensing and Spatial Information Sciences. Vol. XXXVII. Part B8. Beijing, China, pp. 685-688.

Hostache, R., Lai, X., Monnier, J., Puech, C., 2010. Assimilation of spatially distributed water levels into a shallow-water flood model. Part II: Use of a remote sensing image of Mosel River. J Hydrol 390 (3-4), 257-268.

Hostache, R., Matgen, P., Schumann, G., Puech, C., Hoffmann, L., Pfister, L., 2009. Water level estimation and reduction of hydraulic model calibration uncertainties using satellite SAR images of floods. IEEE T. Geosci. Remote 47 (2), 431-441.

Kim, B., Tinin, M., 2009. The association of the residual error of dualfrequency global navigation satellite systems with ionospheric turbulence parameters. J. Atmos. Sol. Terr. Phys. 71 (17-18), $1967-$ 1973.

Lai, X., Monnier, J., 2009. Assimilation of spatially distributed water levels into a shallow-water flood model. Part I: Mathematical method and test case. J Hydrol 377 (1-2), 1 - 11.

Madsen, H., Skotner, C., 2005. Adaptive state updating in real-time river flow forecasting - a combined filtering and error forecasting procedure. J. Hydrol. 308, 302-312.

Martinez, J., Martinez, M., Garcia-Cerezo, A., 2000. A new method of generating differential GPS corrections. Control Eng. Pract. 8 (3), $253-258$.

Matgen, P., Montanari, M., Hostache, R., Pfister, L., Hoffmann, L., Plaza, D., Pauwels, V. R. N., De Lannoy, G. J. M., De Keyser, R., Savenije, H. H. G., 2010. Towards the sequential assimilation of SAR-derived water stages into hydraulic models using the particle filter: proof of concept. Hydrol. Earth Syst. Sci. 14, 1773-1785.

Montanari, M., Hostache, R., Matgen, P., Schumann, G., Pfister, L., Hoffmann, L., 2009. Calibration and sequential updating of a coupled hydrologic-hydraulic model using remote sensing-derived water stages. Hydrol. Earth Syst. Sci. 13, 367-380.
Moradkhani, H., 2007. Hydrologic remote sensing and land surface data assimilation. Sensors 8, 2986-3004.

Neal, J. C., Atkinson, P. M., Hutton, C. W., 2007. Flood inundation model updating using an ensemble kalman filter and spatially distributed measurements. J. Hydrol. 336, 401-415.

Pappenberger, F., Frodsham, K., Beven, K., Romanowicz, R., Matgen, P., 2007. Fuzzy set approach to calibrating distributed flood inundation models using remote sensing observations. Hydrol. Earth Syst. Sci. 11, 739-752.

Plaza, D. A., De Keyser, R., De Lannoy, G. J. M., Giustarini, L., Matgen, P., Pauwels, V. R. N., 2012. The importance of parameter resampling for soil moisture data assimilation into hydrologic models using the particle filter. Hydrol. Earth Syst. Sci. 16 (2), 375-390.

Smith, P., Hughes, D., Beven, K., Cross, P., Tych, W., Coulson, G., Blair, G., 2009. Towards the provision of site specific flood warnings using wireless sensor networks. Meteorol. Appl. 15 (1), 5764.

Smith, P. J., Beven, K. J., Tawn, J. A., 2008. Detection of structural inadequacy in process-based hydrological models: A particlefiltering approach. Water Resour. Res. 44, W09403.

USACE, 2002. United States Army Corps of Engineers: Theoretical basis for one-dimensional flow calculations. In: Hydraulic reference manual. USACE, Davis (CA, USA), Ch. 2, version 3.1 URL http: //www.hec.usace.army.mil/software/hec-ras/

Watson, C., Coleman, R., Handsworth, R., 2008. Coastal tide gauge calibration: A case study at macquarie island using gps buoy techniques. Journal of Coastal Research 24 (4), 1071-1079.

Yoon, Y., Durand, M., Merry, C. J., Clark, E. A., Andreadis, K. M., Alsdorf, D. E., 2012. Estimating river bathymetry from data assimilation of synthetic swot measurements. J Hydrol 464$465(0), 363-375$. 